\title{
Advances in Probing Wood-Coating Interface by Microscopy: A Review
}

\author{
Adya P. Singh ${ }^{1 *}$, Byung-Dae Park ${ }^{1}$, Arif Nuryawan ${ }^{1}$, Menda Kazayawoko ${ }^{2}$ \\ ${ }^{1}$ Department of Wood Science and Technology, Kyungpook National University, Daegu, South Korea; ${ }^{2}$ Departement de Gestion des \\ Ressources Naturelles, Faculte des Sciences Agronomiques, Universite de Kinshasa, Kinshasa, Democratic Republic of the Congo. \\ Email: *adyasingh@hotmail.com
}

Received December $18^{\text {th }}$, 2012; revised January $20^{\text {th }}$, 2013; accepted January $28^{\text {th }}, 2013$

\begin{abstract}
Surface coatings provide protection to wood products against weathering and other deteriorating factors, such as moisture uptake and microbial invasion. The effectiveness of coatings depends on many factors, including how well the applied coatings adhere to the wood surface. Coating adhesion to wood involves both chemical and physical interactions between the coating and wood tissues in contact, and the particular focus of this mini-review will be on the advances being made in understanding the physical aspects of the interaction by probing wood-coating interface using novel and high resolution imaging techniques, including confocal laser scanning microscopy (CLSM), SEM-backscattered electron imaging and correlative microscopy employing light, confocal and scanning electron microscopy.
\end{abstract}

Keywords: Wood-Coating Interface; Coating Adhesion; Correlative Microscopy; Backscattered Electron Imaging; Confocal Laser Scanning Microscopy

\section{Introduction}

Wood products exposed in service under outdoor conditions can deteriorate from the damaging effects of solar radiation, which can cause weathering of cell walls [1$10]$, and cell wall degradation by microorganisms $[11,12]$. Application of suitable coatings to the product surface can significantly extend the service life by preventing solar radiation from reaching the wood surface and organisms and moisture to come in direct contact with the underlying wood tissues. Among the factors that influence coating performance coating adhesion to wood is of major importance [13], which primarily depends on coating penetration into wood [14] and mechanical anchorage through entanglement of coatings within penetrated surface and subsurface layers of wood tissues $[15,16]$. Examining wood-coating interface is therefore important to understand the nature of wood-coating interaction, information that can be helpful in evaluating adhesion and performance of applied coatings and ultimately in optimizing formulations and processes to achieve high durability of wood-coating composites.

Microscopy has been the most widely applied tool to examine and image wood-coating interface. Whereas the majority microscopic studies have aimed to record information on depth to which the applied coatings can penetrate into wood products made from different wood

\footnotetext{
"Corresponding author.
}

types as well as those variously surfaced [14,17-24], specialized microscopic techniques have also been developed and employed that can resolve wood-coating interface more clearly, providing improved understanding of wood-coating interaction at cellular and subcellular levels $[15,16,25]$. In this mini-review the focus will be on these technical advances in probing woodcoating interface, often involving a combination of different types of microscopes that differ in their imaging capabilities and can provide complementary information. The information presented is in a broader context to include places of physical contact of coatings with the surfaces of wood products to which they are applied as well as all contact interfaces between penetrated coatings and cell walls around cell lumens and lining cell wall cracks within tissue layers underneath the surface.

\section{Novel Microscopic Approaches for Imaging Wood-Coating Interface}

The importance of coating adhesion for coating durability and performance has been recognized for a long time. Among the factors that influence coating adhesion to wood surface, coating penetration into and entanglement within surface and subsurface tissues are important, as improvements in water repellency result in greater dimensional stability and less cracking of wood in outdoor exposures, aspects that have been emphasized in several 
studies $[14,23,24,26]$. The importance of capillary penetration, i.e. coating penetration into larger pores within wood tissues such as lumens of vessels, tracheids and rays, can perhaps be best appreciated from the visual appearance of microscopic images illustrated in a study that combined light microscopy and transmission electron microscopy to understand the mechanism of failure of a clear coating applied to radiata pine (Pinus radiata) wood panels [9]. In this outdoor exposure study the clear coating had failed due mainly to wood failure, involving cell separation in the middle lamella region, resulting from preferential degradation of lignin by UV radiation [4]. Coating adhesion with underlying wood tissues had not been compromised within the period of exposure, judging by the presence of outer layers of intact wood tissues attached to separated segments of the coating. Even in regions showing partial film detachment, the coating film was continuous with the coating material present in rays, resisting coating detachment in contact regions. Further advances in understanding wood-coating interactions have come from the development and use of novel microscopic approaches individually and in combination, which is the main focus of this review. The review is based on others' work as well as author's own investigations.

\section{Imaging of Wood-Coating Interface by Confocal Laser Scanning Microscopy (CLSM)}

CLSM is proving as an important information tool in many areas of biological research because of its capabilities in combining non-destructive optical sectioning through relatively thick samples and 3-D reconstruction based on images produced from sequential optical sections. This eliminates the need for using chemical fixation and polymer embedding for serial sectioning, which can alter cellular structures and is a time-consuming and tedious way of obtaining information from a large volume of tissues. CLSM also produces sharper images than achievable by other optical microscopes, because with the help of an aperture out-of-focus fluorescence is largely eliminated, which results in an increase in contrast, image clarity and detection sensitivity. Additionally, because of its capabilities for live cell imaging, in cell biological, physiological and molecular studies CLSM is proving to be a highly desirable tool for obtaining spatial and temporal information at high resolution, making it possible to understand the intricacies of such vital processes as molecular and organelle dynamics, intra- and intercellular communication and signal perception and processing.

In wood coatings area, a study [15] comparing CLSM and light microscopy (LM) to image the interface between $P$. radiata wood and a clear coating showed
CLSM to be markedly superior to LM in obtaining information on the physical nature of interaction between the coating and the surface and outermost subsurface tissue layers (Figure 1). Whereas the contact interface appeared 'fuzzy' in LM images, CLSM clearly resolved the interface, enabling coating penetration into fine cracks, which apparently formed during the surfacing of wood with planer knives, to be clearly visualized. As the presence of small size cracks can greatly increase the cell wall surface area for contact with applied coatings, and thus enhancing coating adhesion through mechanical interlocking, it is important to develop and use micro-
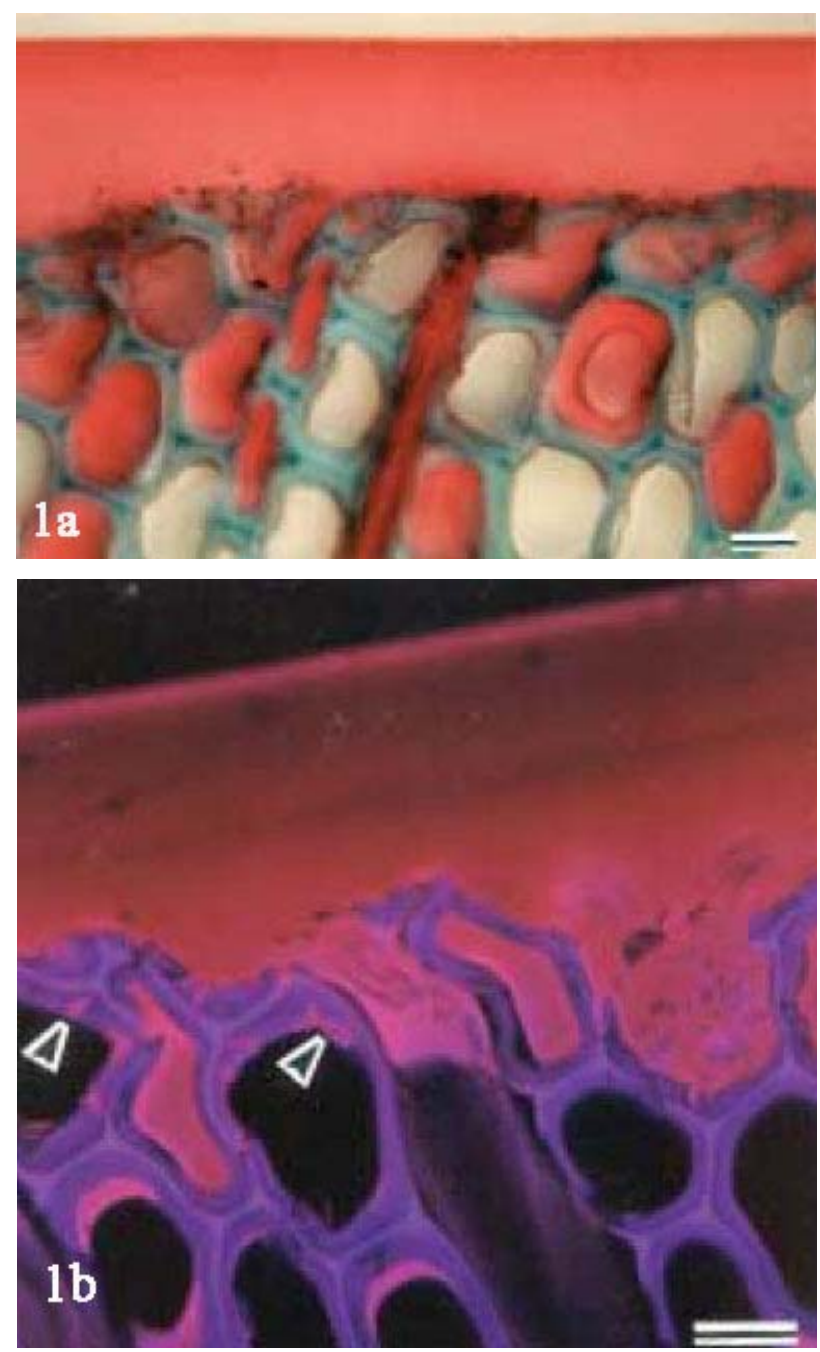

Figure 1. (a) LM view of wood-coating interface. The presence of coating material (red color) in cell lumens can be resolved but not its penetration into cracks within cell walls (light blue color). Bar $=20 \mu \mathrm{m}$. The micrograph is reproduced from JCT Research, Vol. 1, No. 3, 2004; (b) CLSM view of wood-coating interface. The presence of coating material (crimson color) within cell wall (purple color) cracks (arrowheads) can be clearly resolved. Bar $=20 \mu \mathrm{m}$. The micrograph is reproduced from JCT Research, Vol. 1, No. 3, 2004. 
scopic techniques that not only provide information on coating penetration into wood microcapillaries, such as lumens of vessels, tracheids and rays [23] but can also reveal penetration into cell wall cracks formed during the surfacing of wood products. Although scanning electron microscopy (SEM) and transmission electron microscopy (TEM) offer greater resolution compared to CLSM, imaging of wood-coating interface using such tools requires special preparation techniques to visualize coatings [25] and involve rather labor-intensive and time-consuming processing of samples [5]. CLSM can provide high resolution images within a relatively short time, particularly when images are captured in one plane from a single optical slice, and thus has the potential for wide applications in wood coatings research.

\section{Correlative Microscopy in Wood Coatings Research}

While LM [23], CLSM [15] and SEM [14,24] have provided valuable understanding of coating penetration into wood, which can be related to coating adhesion, more novel technical approaches, such as correlative microscopy [16] and X-ray imaging and analysis [27] are providing more complete information on wood-coating interaction. Here an example is presented where compareson of LM, CLSM and SEM was made for their capabilities to resolve greatly distorted surface tissues in a $P$. radiata panel that had been band-sawn to produce a highly rough surface texture in order to understand the pattern of distribution of a stain coating within these tissues. In this study [16] $P$. radiata plywood had been saw-textured to prepare a rough surface which was subsequently coated with a film-forming acrylic stain coating. For correlative microscopy, sections were transversely cut across wood-coating interface using a sliding microtome, and stained with toluidine blue, a stain widely used to contrast lignified cell walls [28]. The sections were initially examined by LM, which was useful in screening the sections to capture a large number of images of the highly irregular surface with greatly distorted tissues to compare earlywood and latewood tissues for micromorphological differences and in the extent of distortions. However, LM proved inadequate in clearly resolving individual cells and differentiating cell walls from cell lumens particularly in the highly distorted tissues. Using LM alone it was therefore not possible to clearly trace penetration pathways of the applied coating and the pattern of its distribution within distorted surface tissues. Same sections were then examined sequentially by LM, CLSM and SEM, imaging the same tissue region, to compare imaging capabilities of the three different microscope systems in resolving distorted tissues with a view to obtain more complete information on coating penetration and distribution (Figure 2).
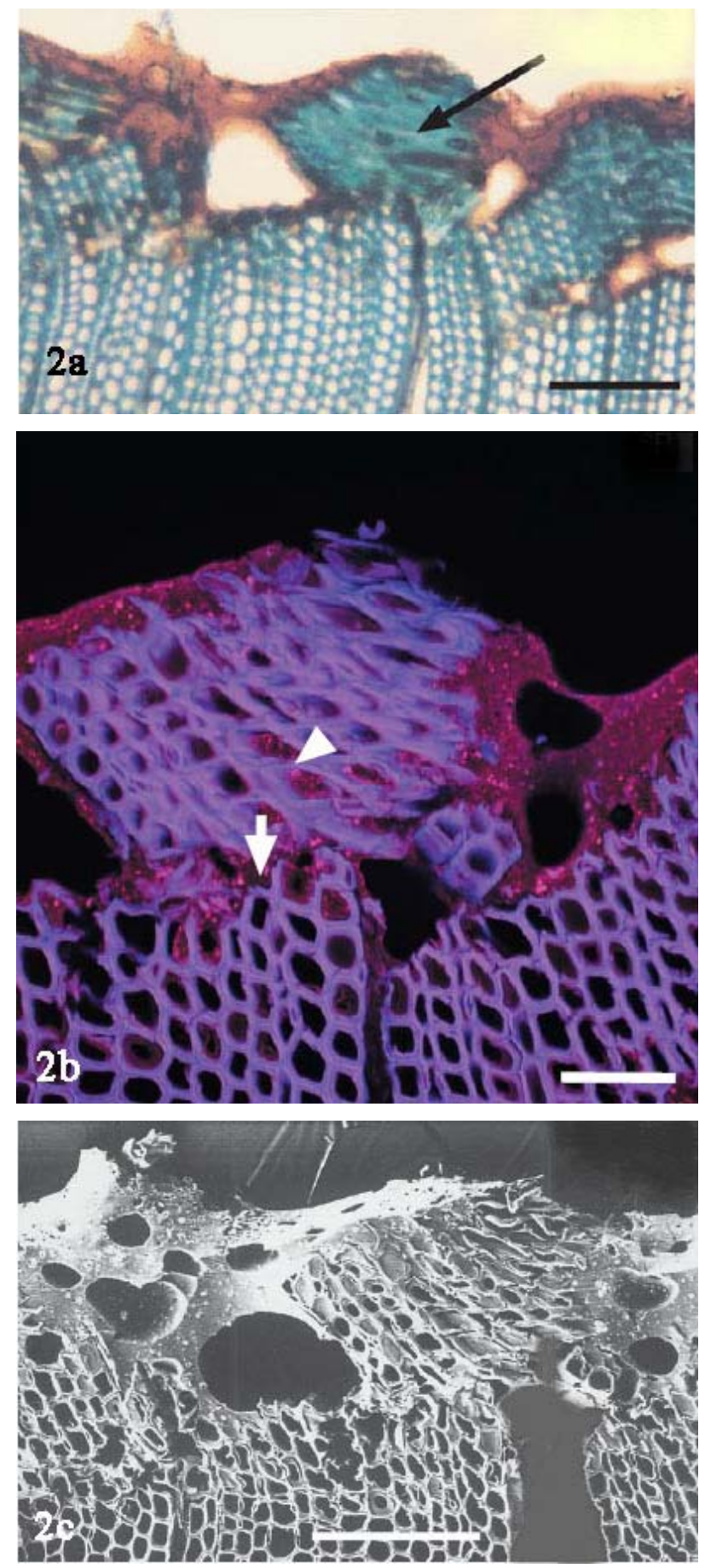

Figure 2. LM (a), CLSM (b) and SEM (c) micrographs of the same section through the coated face of a saw-textured plywood. The distorted surface tissues (arrow) appear fuzzy in the LM image (a) but the same tissue mass is clearly resolved in CLSM (b) and SEM (c) images. In (b) the presence of coating material in cell lumens (arrowhead) and within a crack across the cell walls (arrow) is resolvable. The presence of the coating material in cell lumens is also resolvable in (c) but not as clearly as in (b). Bar in (a) $=\mathbf{2 0 0}$ $\mu \mathrm{m}$; bar in (b) $=100 \mu \mathrm{m}$; bar in (c) $=200 \mu \mathrm{m}$. All micrographs in this figure are reproduced from JCT Research, Vol. 3, No. 3, 2006. 
The surface tissue region examined correlatively and sequentially by LM, CLSM and SEM was extremely distorted resulting from twisting of tissues relative to the plane of the underlying base tissues. The depth into the tissues from which information can be extracted using LM is very limited compared to CLSM or SEM. Thus, whereas the twisted tissue region appeared 'fuzzy' under $\mathrm{LM}$, the features of individual cells within this tissue mass were clearly resolved by both CLSM and SEM. CLSM proved most suitable, as the brilliant color contrast differentiation achieved between the coating and cell walls enabled the intricate pattern of coating penetration and distribution within the distorted tissue mass to be clearly visualized. In addition to coating penetration of cell lumens, the presence of coating within the small size cell wall cracks formed during band-sawing of the panel surface was confirmed, which helps explain the observed excellent outdoor performance of a similar texture-coating system [29].

The example of the work employing correlative microscopy presented here demonstrates the value of obtaining information at cell and cell wall levels. In addition to coating penetration into cell lumens, coating penetration into smaller size gaps and spaces (such as cell wall cracks) within the surface and subsurface tissues is important for effective coating anchorage and thus coating adhesion to wood [30]. This is particularly relevant to highly textured wood surfaces where rather fragile surface tissues have to be stabilized through enhanced coating absorption and effective coating penetration.

\section{SEM-Backscattered Electron Imaging of Wood-Coating Interface}

The majority studies employing SEM and associated techniques have primarily aimed to assess the depth of coating penetration [14,20,22-24]. SEM is a high resolution imaging tool wih the capability of providing cellular and subcellular level information. However, one major disadvantage with SEM in coatings research is that coatings and wood tissues are not well differentiated because of a similar grayish appearance.

Here we examine a study where SEM-backscattered technique was employed to greatly enhance the contrast of a coating material against the cell walls of penetrated wood tissues, enabling the pathways of coating penetration and the pattern of coating distribution to be clearly resolved (Figure 3). This study used a wood panelcoating system similar to that described in the section 4 of this paper. Saw-textured radiata pine plywood panels were coated with a oil modified stain. Sections cut transversely through the wood-coating interface were treated with osmium tetroxide, a heavy metal stain that reacted with the oil borne coating but not with wood cell walls.
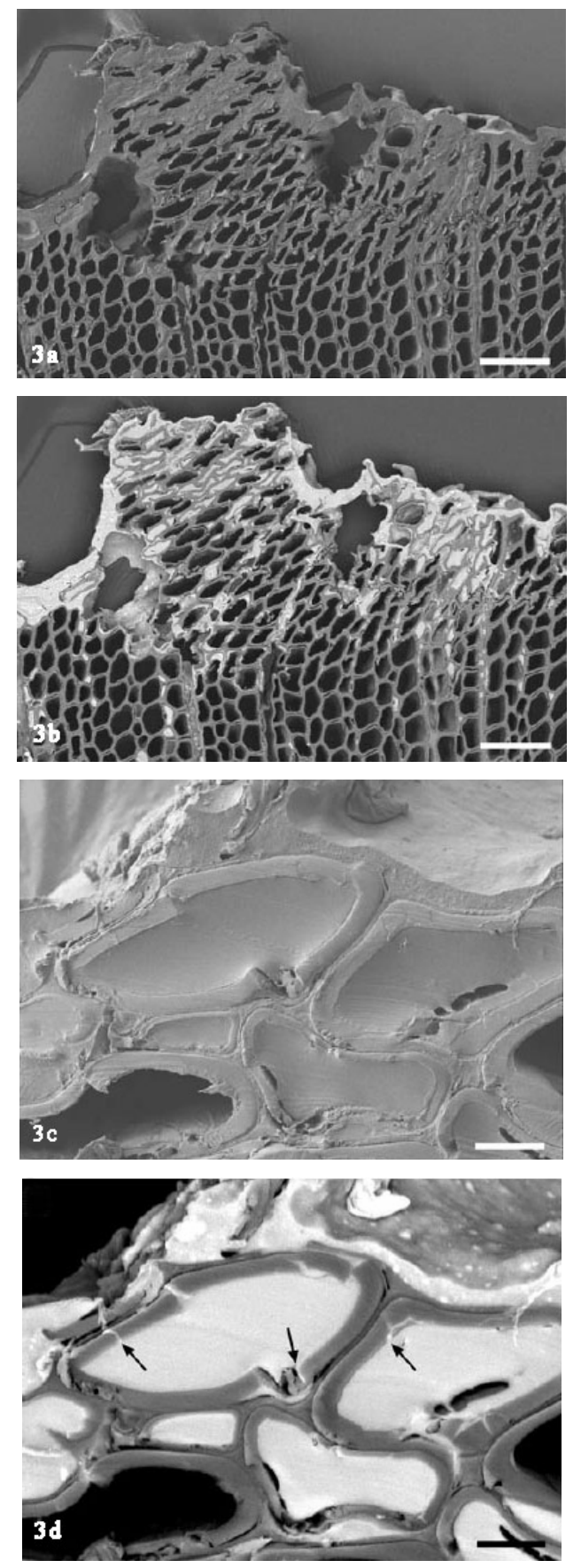

Figure 3. (a), (b) FE-SEM micrographs of the same section taken in secondary electron (a) and backscattered electron (b) imaging modes. In (a) the coating material is poorly differentiated, from wood cell walls because of a similar contrast, but in (b) the bright appearing coating material is clearly resolved. Bars $=100 \mu \mathrm{m}$; (c), (d) High magnification FE-SEM micrographs of the same tissue region in a section taken in secondary electron (c) and backscattered electron (d) imaging modes. In (c) the coating material is poorly differentiated from cell walls, but in (d) the presence of bright appearing coating material within cell lumens and fine cell wall cracks (arrows) is clearly resolved. Bars $=10$ $\mu \mathrm{m}$. All micrographs in this figure are reproduced from JCT Research, Vol. 4, No. 2, 2007. 
The osmium treated sections were first imaged with SEI (secondary electron imaging) mode of a FE-SEM (field emission scanning electron microscope), and the same sections were then viewed under BEI (backscattered electron imaging) mode. Comparison of the two imaging modes using the same sections showed a distinct advantage of the BEI imaging over the SEI imaging. Whereas in the SEI mode the coating was poorly differentiated from wood tissues, in the BEI mode the coating material appeared bright and thus was readily differentiated from wood tissues, which enabled the intricate pathway of coating penetration and distribution within the highly distorted saw-textured surface tissues to be readily examined, including penetration of the coating material into very small size cell wall cracks present within these tissues. Osmium tetroxide staining of the coating material was a critical step in the process.

Backscattered electron imaging provides information on the presence and location of high atomic number substances within a specimen, which appear bright under this imaging mode due to high yields of backscattered electron signals, and this was the basis for coupling the heavy metal osmium tetroxide with the coating. Osmium tetroxide has been used previously as a stain or tracer in other systems involving backscattered electron imaging [31,32], but in the work described above it was the first time backscattered electron imaging was used to undertake high resolution imaging work on the penetration and distribution of a coating into wood. Similar approach can also work for examining other coating systems with wood tissues, provided suitable high atomic number substances can be employed that can specifically react with the coatings of interest, and without affinity for wood cell walls.

\section{Conclusion}

While light microscopy has been useful in determining the depth of coating penetration into wood, it is not suitable for examining wood-coating interface because of its inability to clearly resolve particularly the interface between the outermost surface layer of wood tissues and the applied coating. The novel and more specific microscopy approaches employed in more recent years are proving valuable in wood-coating interaction studies, particularly involving rough-texture wood surfaces containing highly distorted tissues.

\section{Future Prospects}

The technical advances discussed in imaging woodcoating interface are providing greater understanding of the physical aspects of wood-coating interaction. Combined use of microscopy techniques with complementary capabilities can provide more complete information, and in future wood-coating studies the scope of this approach should expand.

The majority microscopic studies undertaken have mainly focused on examining depth of coating penetration. While understanding of this is important in the context of evaluation of coating adhesion, higher resolution imaging should be combined to understand particularly the effect of surface preparation on wood tissues at finer levels. As documented by CLSM [15], even relatively smooth surfacing with planer knives can generate cracks of varying dimensions within cell walls, which collectively can significantly increase cell wall surface areas, thus enhancing the anchorage of penetrated coating material. While LM can still serve as the basic imaging tool for rapid assessment of coating penetration and distribution, the scope in future studies should widen to include the assessment using also tools that can provide 3-D imaging capability and high resolution, such as CLSM, FE-SEM and TEM, which in addition to revealing the finer aspects of wood-coating interaction can provide useful information for assessing coating performance, such as demonstrated in a study employing TEM [5].

\section{Acknowledgements}

This work was supported by the Korean Ministry of Education, Science and Technology and the Korean Federation of Science and Technology Societies.

\section{REFERENCES}

[1] R. M. Rowell, W. C. Feist and W. D. Ellis, "Weathering of Chemically Modified Southern Pine," Wood Science, Vol. 13, No. 4, 1981, pp. 202-208.

[2] D. N.-S. Hon, "Weathering Reactions and Protection of Wood Surfaces,” Journal of Applied Polymer Science, Vol. 37, No. 2, 1983, pp. 845-864.

[3] W. C. Feist, R. M. Rowell and J. Youngquist, "Weathering and Finish Performance of Acetylated Aspen Fiberboard," Wood and Fiber Science, Vol. 23, No. 2, 1991, pp. 260-272.

[4] M. Kuo and H. N. Hu, "Ultrastructural Changes of Photodegradation of Wood Surfaces Exposed to UV Light," Holzforschung, Vol. 45, No. 5, 1991, pp. 347-353. doi:10.1515/hfsg.1991.45.5.347

[5] A. P. Singh, E. A. Dunningham and D. V. Plackett, “Assessing the Performance of a Commercial Wood Stain by Transmission Electron Microscopy,” Holzforschung, Vol. 49, No. 3, 1995, pp. 255-258.

[6] P. D. Evans, P. D. Thay and K. J. Schmalzl, "Degradation of Wood Surfaces During Natural Weathering. Effect of Lignin and Cellulose and on the Adhesion of Acrylic Latex Primers," Wood Science and Technology, Vol. 30, No. 6, 1996, pp. 411-422. doi:10.1007/BF00244437

[7] D. V. Plackett, E. A. Dunningham and A. P. Singh, "Weathering of Chemically Modified Wood," In: D. N.-S. 
Hon, Ed., Chemical Modification of Lignocellulosic Materials, Marcel Dekker, Inc., New York, 1996, pp. 277294.

[8] R. S. Williams, M. T. Knaebe and W. C. Feist, "Erosion Rates of Wood during Natural Weathering. Part II. Earlywood and Latewood Erosion Rates," Wood and Fiber Science, Vol. 33, No. 1, 2001, pp. 43-49.

[9] A. P. Singh and B. S. W. Dawson, "The Mechanism of Failure of Clear Coated Wooden Boards as Revealed by Microscopy,” IAWA Journal, Vol. 24, No. 1, 2003, pp. 111.

[10] P. D. Evans, "Weathering of Wood and Wood Composites,” In: R. M. Rowell, Ed., Handbook of Wood Chemistry and Wood Composites, 2nd Edition, CRC Press, Taylor and Francis Group, Boca Raton, 2013, pp. 151-216.

[11] R. A. Blanchette, T. Nilsson, G. F. Daniel and A. R. Abad, "Biological Degradation of Wood," In: R. M. Rowell and J. Barbour, Eds., Archaeological Wood: Properties, Chemistry and Preservation, American Chemical Society, Washington DC, 1990, pp. 147-174.

[12] A. P. Singh and J. A. Butcher, "Bacterial Degradation of Wood Cell Walls: A Review of Degradation Patterns," Journal of the Institute of Wood Science, Vol. 12, 1991, pp. 143-157.

[13] R. S. Williams and W. C. Feist, "Durability of Paint or Solid-Color Stain Applied to Preweathered Wood," Forest Products Journal, Vol. 43, No. 1, 1993, pp. 8-14.

[14] M. de Meijer and H. Militz, "Wet Adhesion of Low-VOC Coatings on Wood: A Quantitative Analysis," Progress in Organic Coatings, Vol. 38, No. 3-4, 2000, pp. 223-240. doi:10.1016/S0300-9440(00)00108-9

[15] A. P. Singh and B. S. W. Dawson, "Confocal Microscope-A Valuable Tool For Examining Wood-Coating Interface,” Journal of Coatings Technology and Research, Vol. 1, No. 3, 2004, pp. 235-237. doi:10.1007/s11998-004-0017-z

[16] A. P. Singh and B. S. W. Dawson, "Microscopic Assessment of the Effect of Saw-Textured Pinus radiata Plywood Surface on the Distribution of a Film-Forming Acrylic Stain,” Journal of Coatings Technology and Research, Vol. 3, No. 3, 2006, pp. 193-201. doi:10.1007/BF02774508

[17] M. H. Schneider and W. A. Côté, "Studies of Wood and Coating Interactions Using Fluorescence Microscopy and Pyrolysis Gas-Liquid Chromatography,” Journal of Paint Technology, Vol. 39, No. 511, 1967, pp. 465-471.

[18] W. A. Côté and R. G. Robinson, “A Comparative Study of Wood-Coating Interaction Using Incident Fluorescence and Transmitted Fluorescence Microscopy," Journal of Paint Technology, Vol. 40, No. 525, 1968, pp. 427-432.

[19] M. H. Schneider, "Coating Penetration into Wood Substance Studied with Electron Microscopy Using Replica Techniques,” Journal of Paint Technology, Vol. 42, No. 547, 1970, pp. 457-460.
[20] M. H. Schneider, "Scanning Electron Microscope Study of a Coating Component Deposited from Solution into Wood," Journal of the Oil and Color Chemists Association, Vol. 62, No. 11, 1979, pp. 441-444.

[21] M. H. Schneider, "Microscopic Distribution of Linseed Oil after Application to Wood Surface,” Journal of Coatings Technology, Vol. 52, No. 665, 1980, pp. 64-67.

[22] R. M. Nussbaum, "Penetration of Water-Borne Alkyd Emulsions and Solvent Borne Alkyds into Wood," Holz als Roh-und Werkstoff, Vol. 52, No. 6, 1994, pp. 389-393. doi:10.1007/BF02615391

[23] M. de Meijer, K. Thurich and H. Militz, "Comparative Study on Penetration Characteristics of Modern Wood Coatings,” Wood Science and Technology, Vol. 32, No. 5, 1998, pp. 347-365. doi:10.1007/BF00702791

[24] R. E. Hernández and J. Cool, "Evaluation of Three Surfacing Methods on Paper Birch Wood in Relation to Water- and Solvent-Borne Coating Performance," Wood and Fiber Science, Vol. 40, No. 3, 2008, pp. 459-469.

[25] A. P. Singh, A. Ratz and B. S. W. Dawson, "A Novel Method for High Resolution Imaging of Coating Distribution within a Rough Textured Plywood Surface,” Journal of Coatings Technology and Research, Vol. 4, No. 2, 2007, pp. 207-210. doi:10.1007/s11998-007-9019-y

[26] L. F. de Moura and R. E. Hernández, "Evaluation of Varnish Coating Performance for Two Surfacing Methods on Sugar Maple Wood,” Wood and Fiber Science, Vol. 37, No. 2, 2005, pp. 355-366.

[27] J. Van den Bulcke, M. Boone, J. Van Acker and L. Van Hoorebeke, "High-Resolution X-Ray Imaging and Analysis of Coatings on and in Wood," Journal of Coatings Technology and Research, Vol. 7, No. 2, 2010, pp. 271277. doi:10.1007/s11998-009-9182-4

[28] T. P. O’Brien and M. E. McCully, "Plant Structure and Development: A Pictorial and Physiological Approach,” The Macmillan Company/Collier-Macmillan Ltd., London, 1969.

[29] R. S. Williams and W. C. Feist, "Effect of Preweathering, Surface Roughness and Wood Species on the Performance of Paints and Stains," Journal of Coatings Technology, Vol. 66, No. 828, 1994, p. 109.

[30] B. S. W. Dawson, S. Gallagher and A. P. Singh, "Microscopic View of Wood and Coating Interaction and Coating Performance on Wood,” Forest Research Bulletin, No. 228, New Zealand Forest Research Institute, Rotorua, 2003.

[31] P. B. DeNee and R. L. Carpenter, "Application of Heavy Metal Staining $\left(\mathrm{O}_{\mathrm{s}} \mathrm{O}_{4}\right)$ /Backscattered Electron Imaging Technique to the Study of Organic Aerosols," Proceedings of the 14th Annual Conference of the Microbeam Analysis Society, San Antonio, 1979, pp. 8-10.

[32] D. E. Schraufnagel and D. P. Ganesan, "Tracers in Vascular Casting Resins Enhance Backscattering Brightness,” Scanning Microscopy, Vol. 12, No. 4, 1998, pp. 631-639. 